\title{
BILATERAL DISLOCATION OF THE TALUS OF UNKNOWN ETIOLOGY
}

\author{
M. K. Jensen, Hillerød, Denmark
}

A woman aged sixty-four attended for treatment of congestive heart failure, and during the clinical examination it was noticed that both feet were markedly deformed. The patient said that her feet had been normal until she was forty-nine, when she began to notice swelling of both ankles, alteration of the shape of the feet, and moderate pain. There had been no injury. The pain ceased in the course of six months, but the deformity continued to increase for five or six years. Thereafter the feet caused no inconvenience except for difficulty in fitting shoes.
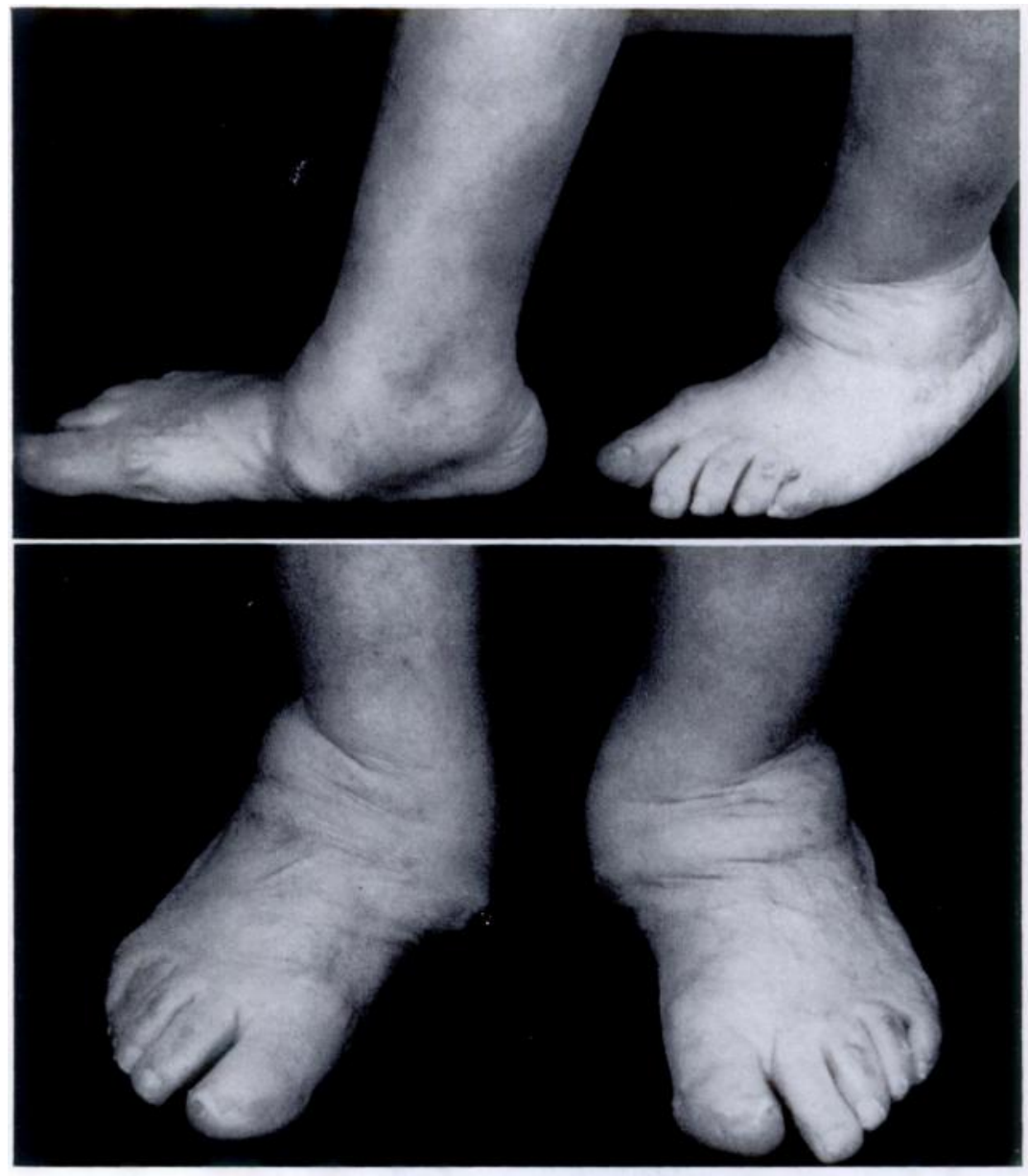

FIC. 1

Photographs of the feet. Note the medial prominence of the talus.

On examination there was on each side pronounced deformity in the tarsal region with prominence of the talus at the medial border of the foot, seemingly due to lateral displacement of the calcaneum and the navicular bone (Fig. 1). The range of ankle movement was reduced to about 10 or 15 degrees, and movement at the subtalar joint was absent. Walking with the feet bare was awkward because weight was transmitted to the inner border of each foot, 
but with arch supports the patient was able to walk with hardly any discomfort. The shoes were bulged by the prominent tali. Examination of other joints showed hyperextensibility of the metacarpo-phalangeal joints and slight hyperextension at the knees. There was slightly increased antero-posterior mobility of both tibiae upon the femora.

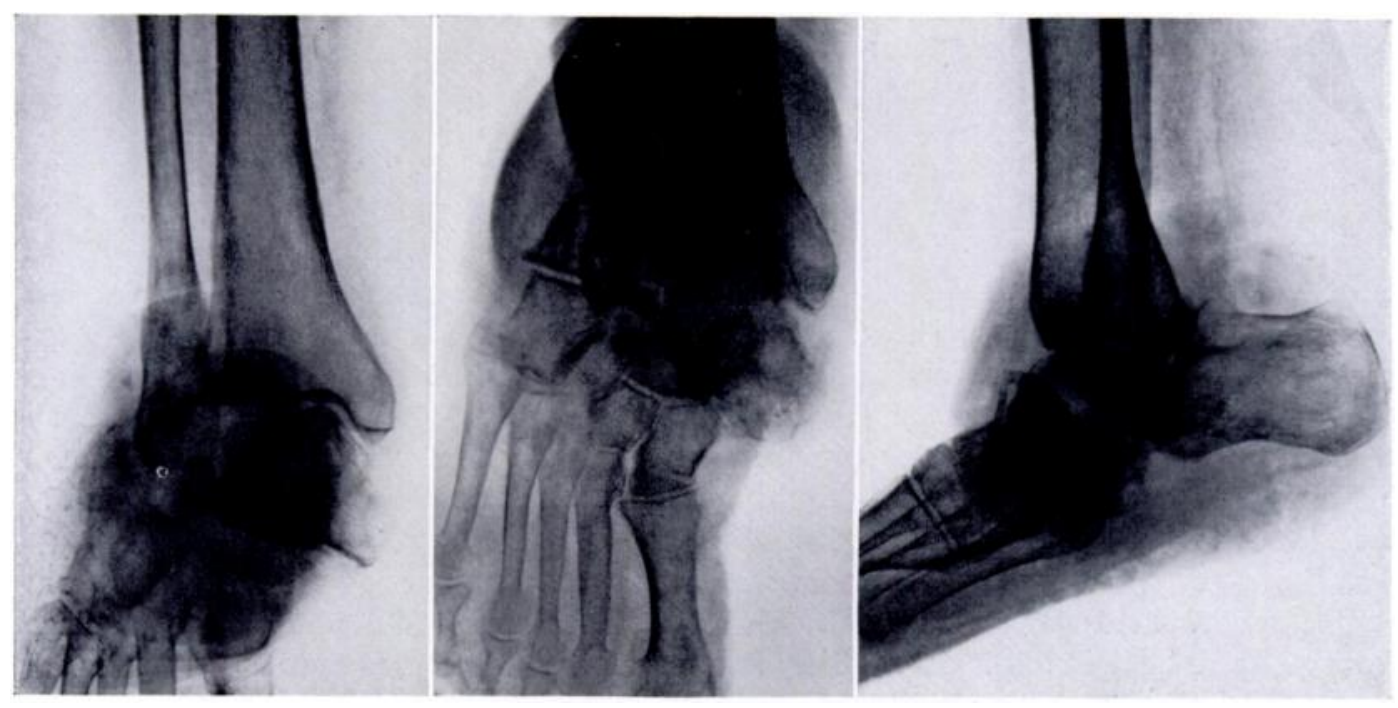

FIG. 2

Radiographs of the right ankle and foot. The left foot was similar.

On general examination, apart from the cardiac condition, nothing abnormal was found. There was no evidence of hyperelasticity of the skin. Neurological examination failed to reveal any abnormality: sensibility to pain, touch and temperature were normal, as were the vibration sense, the reflexes and the Romberg test. Normal arterial pulses were present in the feet. The Wassermann reaction was negative in the blood and the cerebrospinal fluid.

Radiographic examination-Radiographs of the feet showed, on each side, dislocation of the talo-calcaneal and talo-navicular joints, so that the bones distal to the talus were displaced laterally and into marked valgus (Fig. 2). The distal articular surface of the talus, which should articulate with the navicular bone, lay subcutaneously at the medial side of the foot. The lower end of the fibula abutted against the front of the calcaneum: on the left side the distal end of the fibula showed a spur of new bone. The navicular bones on both sides were wedge-shaped, with the apex of the wedge directed medially. Radiographs of the knees showed osteoarthritis and hyperextension of the joints. Radiographs of the other joints and of the spine showed nothing abnormal.

Comment - I have not previously heard of a similar foot deformity arising without injury and I have been unable to find any reference to it in the literature. The cause is completely unknown. Just possibly it might be related to ligamentous laxity and hypermobility, because abnormal laxity was present in the metacarpo-phalangeal joints and slightly in the knees. But similar laxity has been seen many times before without complications. 